\title{
Traduire
}

Une eutre perspective sur $r$ tatadciction

Revue française de la traduction

$240 \mid 2019$

Quand la politique s'en mêle

\section{Lu pour vous : La Langue de Trump, de Bérengère Viennot}

\section{Christine Cross}

\section{(2) OpenEdition}

1 Journals

Édition électronique

URL : http://journals.openedition.org/traduire/1662

DOI : 10.4000/traduire.1662

ISSN : 2272-9992

Éditeur

Société française des traducteurs

\section{Édition imprimée}

Date de publication : 20 juin 2019

Pagination : 45-47

ISSN : 0395-773X

\section{Référence électronique}

Christine Cross, « Lu pour vous : La Langue de Trump, de Bérengère Viennot », Traduire [En ligne], 240 |

2019, mis en ligne le 20 juin 2019, consulté le 25 septembre 2020. URL : http://

journals.openedition.org/traduire/1662 ; DOI : https://doi.org/10.4000/traduire.1662 


\section{Lu pour vous}

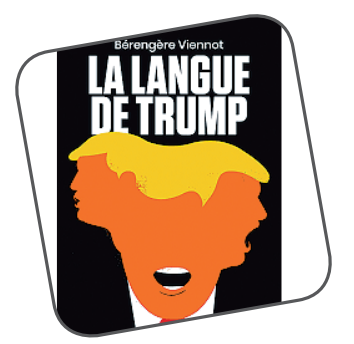

\section{La Langue de Trump, de Bérengère Viennot}

\section{Christine Cross}

Dans un numéro dont la thématique est "Quand la politique s'en mêle», Traduire ne pouvait passer à côté d'un ouvrage publié en janvier 2019, signé Bérengère Viennot et intitulé La Langue de Trump.

Vaste sujet, direz-vous, et à raison. En réalité, ce témoignage dépasse les aspects purement linguistiques et offre un éclairage sur certaines évolutions marquantes de notre époque.

Bérengère Viennot traduit pour la presse depuis bientôt vingt ans. C'est une professionnelle aguerrie qui a l'habitude du discours enlevé, de la langue châtiée, des tournures de phrase élégantes, qui sont souvent l'apanage du monde politico-diplomatique.

Dès le début de son ouvrage, l'auteure fait part du choc psychologique qu'a représenté pour elle l'arrivée au pouvoir du $45^{e}$ président des États-Unis. Ce personnage brut de décoffrage est aux antipodes de ses prédécesseurs, dont les textes lui permettaient de faire usage de sa plus belle plume, une satisfaction en soi pour une amoureuse de la langue.

Désormais, changement de décor. Adieu les jolies phrases, bonjour la vulgarité et les entorses à la grammaire et à la syntaxe. La déception est immédiate et difficile à encaisser. Nous avons tous en mémoire les propos maladroits, sexistes et réducteurs à l'égard de Brigitte Macron ("You're in such good shape») dont le sous-texte, des plus explicites, laissait bien entendre: « Vu votre âge, je m'attendais à bien pire!» 
En outre, Bérengère Viennot nous signale que le langage de Trump aurait même baissé d'un (voire plusieurs) cran(s) depuis sa prise de fonction. Là où, pendant la campagne électorale, il lui arrivait de prononcer des phrases entières, l'heure est désormais à l'appauvrissement du discours, au massacre des règles les plus élémentaires. Et tout cela pour laisser place à une communication sans filtre et souvent incohérente!

Le défi ne consiste plus à trouver des formules suffisamment élégantes pour transposer un tel discours, mais à parvenir (jusqu'à quel point si l'on veut éviter de choquer les esprits?) à s'abaisser au niveau de vulgarité, de brutalité et de misogynie d'un Donald Trump. Et cela, sachant que le traducteur, comme nous l'explique à bon escient Bérengère Viennot, est censé être un individu neutre totalement invisible, qui ne laisse jamais percer ses sentiments ni son avis. Agir autrement serait une faute professionnelle, ce qui ne fait qu'exacerber le dilemme!

Début février 2019, j'ai eu l'occasion d'assister à une rencontre organisée par les éditeurs en présence de l'auteure et de Thomas Snégaroff, journaliste et historien, spécialiste des États-Unis et habitué des plateaux de télévision. Le débat a donc porté non seulement sur le niveau du langage "éructé» par l'actuel président américain, mais également sur les raisons de son choix de registre et, plus important encore, sur son adoption des réseaux sociaux comme moyen de communication privilégié.

Le discours est ensuite passé du "simple» examen, exemples à l'appui, des problèmes posés par le non-respect des règles de grammaire et de syntaxe par Donald Trump, à des théories sur le personnage en tant que tel. Thomas Snégaroff a ainsi brossé un portrait peu flatteur du "POTUS'» (ce que fait également Bérengère Viennot dans son écrit). Pour lui, ce n'est pas un hasard si ce produit du monde des médias et de la téléréalité a opté pour un langage simple, réduit à son expression la plus minimaliste, et si Twitter a pris une telle place dans son système de gouvernance.

Face à toutes les questions soulevées par l'auteure et le journaliste politologue, on est également en droit de se demander si l'utilisation très personnelle de ce moyen de communication répond à l'impérieuse nécessité de masquer un manque de

1. POTUS: President of the United States. 
culture, un éventuel illettrisme et/ou un mépris total pour l'expression, le style, le vocabulaire.

Je retiens, tant de cette lecture que du débat sur l'ouvrage et son (anti)héros, l'émergence d'une tendance qui dépasse largement le "phénomène» Trump... tendance qui mérite l'attention des traducteurs actuels et à venir. Car, à persister, elle pourrait sonner le glas de nos belles langues au profit de ce langage codé, lapidaire et peu édifiant, où tout doit être dit en 280 caractères, pas un de plus!

Certes, on pourrait m'objecter qu'une langue, quelle qu'elle soit, a pour rôle de nous permettre de nous comprendre. Dans ce cas, peu importe qu'elle soit exprimée en alexandrins, en prose ou par simple juxtaposition de mots et répétition à l'envi des mêmes vocables (pratique très marquée chez Trump, comme l'a démontré une étude statistique), puisque le message aura été transmis.

Quelle attitude devons-nous alors adopter face à l'évolution de la langue et des techniques de communication, à ce nivellement par le bas? Résister ou subir? Cette baisse de la qualité de la langue est-elle devenue inéluctable? Si l'ouvrage en lui-même m'a un peu laissée sur ma faim par rapport à la promesse de son titre - une analyse en profondeur du langage du $45^{e}$ président des États-Unis -, sa lecture m'aura au moins donné l'occasion de procéder à une réflexion plus large sur les implications pour notre métier de cet emploi à outrance des moyens de communication modernes, contemporains, en ligne.

Bérengère Viennot, La Langue de Trump, Paris, Les Arènes, 2019. 